\title{
Aprotinin Prevents the Development of the Trauma-Induced Multiple Organ Failure in a Chronic Sheep Model ${ }^{1}$ )
}

\author{
Alexander Dwenger ${ }^{1}$, Dierk Remmers ${ }^{2}$, Martin Grotz ${ }^{2}$,Hans-Christoph Pape ${ }^{2}$, Andreas Gruner ${ }^{2}$, Heike Scharff ${ }^{2}$, \\ Marianne Jochum ${ }^{3}$ and Gerd Regel \\ 1 Institut für Klinische Biochemie \\ 2 Unfallchirurgische Klinik \\ Medizinische Hochschule Hannover, Hannover, Germany \\ 3 Abt. für Klinische Chemie und Klinische Biochemie der Chirurgischen Klinik Innenstadt, München, Germany
}

Dedicated to Prof. Dr. Dr. J. Büttner on the occasion of his 65th birthday

Summary: Trauma-induced multiple organ failure in sheep was prevented by aprotinin therapy.

Multiple organ failure was induced in 16 female merino sheep by initial haemorrhagic shock and intramedullary femoral nailing (day 0), and 12 hourly injections of $0.75 \mu \mathrm{g} / \mathrm{kg}$ Escherichia coli endotoxin $+0.7 \mathrm{ml} / \mathrm{kg}$ zymosanactivated plasma (days $1-5)$. In addition, the aprotinin group $(\mathrm{n}=6)$ received simultaneous injections of $5 \mathrm{mg} / \mathrm{kg}$ $(35695 \mathrm{KJU} / \mathrm{kg}$ ) aprotinin, whereas ten animals did not receive aprotinin and served as the control group $(\mathrm{n}=10)$.

Organ functions were monitored for a total of 11 days by measuring haemodynamic, cardio-respiratory and biochemical quantities of blood, urine and epithelial lining fluid.

During the subsequent eleven day period, aprotinin induced a significant $\left({ }^{*} p<0.05\right)$ reduction of the pathological changes (development of multiple organ failure) seen in the control group. Thus, aprotinin prevented an alteration of cardiac function (cardiac index for control/aprotinin groups at day 1: 6.5/6.2, and at day 10: 10.47/7.0* $1 / \mathrm{min} \cdot \mathrm{m}^{2}$ ), an impairment of lung function (mean pulmonary arterial pressure at day $1: 2.26 / 1.86$, and at day 10 : $3.83 / 2.13^{*} \mathrm{kPa}$; epithelial lining fluid/plasma ratio of albumin concentrations as a direct marker of lung capillary permeability damage at day $0: 0.18 / 0.16$, and at day $10: 0.45 / 0.15^{*}$ ), a deterioration of liver function (plasma sorbitol dehydrogenase at day 0:7.9/7.6, and at day 10:29.6/7.4* U/1), but not of renal function (creatinine clearance at day 1: $91.4 / 66.1$, and at day 10: $53.1 / 59.2 \mathrm{ml} / \mathrm{min}$ ). Urinary aprotinin excretion increased up to day 3 , then decreased rapidly despite further aprotinin administration. As a non-specific marker of cell damage, plasma lactate dehydrogenase indicated an aprotinin-induced organ protection (day 0: 501/409, and at day 10: 719/329* U/1).

The neutrophil count and the measured chemiluminescence of neutrophils from the blood and epithelial lining fluid showed that aprotinin reduced the in vivo neutrophil activation, the alveolar neutrophil invasion, the production of inflammatory mediators, and the production of reactive oxygen metabolites during the passage of the capillaryinterstitial-alveolar space by neutrophils.

\section{Introduction}

Neutrophil-mediated pathomechanisms include the production of reactive oxygen metabolites and lysosomal proteinases, leading to organ damage through their effects on blood cascade systems and endothelial cells. This can result in acute respiratory distress, reperfusion injury or multiple organ failure syndromes $(1-8)$. The recently developed model of multiple organ failure in sheep meets all criteria of a human-like neutrophil-associated multiple organ failure syndrome $(9,10)$. The

\footnotetext{
1) This work was supported by the Deutsche Forschungsgemeinschaft (project $\operatorname{Re} 1015 / 1-1$ and /1-2)
}

scavenging of reactive oxygen metabolites can reduce/ prevent the development of those syndromes in animals and humans $(11-13)$. Moreover, the inhibition of proteinases involved in these pathomechanisms can also reduce the effect of inflammatory mediators produced by proteinases of inflammatory cells and by proteinases of the plasma cascade systems (kallikrein/kinin, clotting, fibrinolysis, complement) (14-17). In particular, the inhibition of endotoxin-induced plasma kallikrein and plasmin formation in pigs by high doses of aprotinin has demonstrated the important role of kallikrein- and plasma-induced inflammatory mediator production and their effects in shock pathophysiology (16). Also, in a sheep model with septic shock, the inhibition of kalli- 
krein by aprotinin prevented systemic vasodilatation and pulmonary hypertension (18).

Therefore, our aim was to evaluate whether inhibition of the main target enzymes (plasmin, kallikrein) by aprotinin in a trauma-induced sheep model of multiple organ failure would improve organ functions and prevent multiple organ failure development.

\section{Materials and Methods}

Animal preparation, haemodynamic and cardio-respiratory measurements

All procedures were performed according to the German council's guide for the care and use of laboratory animals of August 18, 1986 and were approved by the animal protection committee of the local government (No. 93600).

Adult female merino sheep were used, weighing $25-30 \mathrm{~kg}$. To produce multiple organ failure, the animals were treated as previously described $(9,10)$. Briefly, at day 0 a venous catheter was placed into the right jugular vein under local anaesthesia and blood was drawn and anticoagulated with sodium citrate for basic measurements. General anaesthesia was induced with $0.5 \mathrm{mg}$ Atropin ${ }^{\circledR}$ and $3.5 \mathrm{mg} / \mathrm{kg}$ body weight Pentobarbital ${ }^{\circledR}$ and was continued by ventilation with halothane/nitrogen oxide/oxygen after intubation. Further catheters were placed into the femoral vein and artery, a Swan-Ganz thermodilution catheter (Baxter model 93 A-131-7F, Edwards Critical Care Division, Irvine, CA, USA) was inserted via an external jugular vein and advanced into the pulmonary artery. Another catheter was located into the urinary bladder (Norta, Modell 10 - No. 9385, BDF Beiersdorf AG, Hamburg, Germany) and connected with a collection tube.

Arterial, central venous, pulmonary arterial and pulmonary capillary wedge pressure were recorded using pressure transducers which were positioned at the level of the right atrium. Calibration was done prior to each measurement. Cardiac index was measured by the thermodilution method. In heparinized arterial and mixed venous blood samples, acidity, carbon dioxide, oxygen tension, and oxygen saturation were recorded using a blood gas analyser (ABL2, Radiometer, Copenhagen, Denmark).

Alveolar-to-arterial oxygen pressure difference, right ventricular stroke work index, pulmonary vascular resistance, and arterio-tovenous oxygen pressure difference were calculated by standard formulae from the quantities measured. Blood (anticoagulated with sodium citrate) for biochemical measurements was drawn every day in the morning before each treatment.

During the experiment the animals did not receive any further operative treatment and had free access to food and water. The animals were observed for a total of 11 days and then sacrificed under anaesthesia by infusion of $0.1 \mathrm{ml} / \mathrm{kg} \mathrm{T61} \mathrm{(Embutramid} \mathrm{+} \mathrm{Mebe-}$ zoniumiodide + Tetracin hydrochloride; Hoechst AG, Frankfurt, Germany).

\section{Bronchoalveolar lavage technique}

Bronchoscopy and bronchoalveolar lavage were performed with a fiberoptic bronchoscope (Olympus BF type 1 T 10, Olympus Corporation of America, New Hyde Park, N.Y., USA) as previously described (19). Under light anaesthesia (1.5 mg/kg thiopental sodium and subsequent injection of $2 \mathrm{mg} / \mathrm{kg}$ pentobarbital) the bronchoalveolar lavage procedure was performed at days 0 (prior to the induction of haemorrhagic shock), day 6 , and day 10 before each medication/treatment.

Haemorrhagic shock and intramedullary femoral nailing

After bronchoscopy blood was drawn in $50 \mathrm{ml}$ portions until the mean arterial pressure was $6.7 \mathrm{kPa}$. This was maintained for 2 hours by fractional blood withdrawal. The total blood volume collected was $450-600 \mathrm{ml}$ (about $20 \mathrm{ml} / \mathrm{kg}$ ). Then, a three-times volume of Ringer-lactate solution and $5 \mathrm{mg} / \mathrm{kg}$ aprotinin $^{2}$ ) in a total of $5 \mathrm{ml}$ of saline were infused (aprotinin was given only in the treatment group) until the mean arterial blood pressure reached a normal level. This was followed by a closed intramedullary femoral nailing procedure with a standard $\mathrm{AO}$ technique (20).

\section{Administration of endotoxin and zymosan-activated} plasma

After overnight recovery, haemodynamic and cardio-respiratory quantities were measured and blood was drawn (anticoagulated with sodium citrate) for the biochemical measurements. Then, 0.7 $\mathrm{ml} / \mathrm{kg}$ zymosan-activated autologous plasma $+0.75 \mu \mathrm{g} / \mathrm{kg}$ endotoxin were injected in $5 \mathrm{ml}$ of saline simultaneously with $5 \mathrm{mg} / \mathrm{kg}$ $(=35695 \mathrm{KIU} / \mathrm{kg}$ ) aprotinin in $5 \mathrm{ml}$ of saline (aprotinin group; $n=6$ ) and without aprotinin (control group; $n=10$ ). This administration was repeated every 12 hours for a total of 5 days, and baseline measurements were performed every day in the morning before each application.

\section{Endotoxin, zymosan-activated plasma and aprotinin preparations}

Escherichia coli endotoxin (serotype 055:B5, Sigma Chemicals, Deisenhofen, Germany) was dissolved in saline and applicated in a total of $5 \mathrm{ml}$ of saline to yield $0.75 \mu \mathrm{g} / \mathrm{kg}$. For the production of zymosan-activated plasma, $450 \mathrm{ml}$ blood (anticoagulated with sodium citrate) were withdrawn at day 0 and centrifuged for 10 min at $800 \mathrm{~g}$ and $22^{\circ} \mathrm{C}$. The plasma was then incubated with sterile zymosan ( $3 \mathrm{mg} / \mathrm{ml}$ plasma) (zymosan A, Sigma Chemicals, Deisenhofen, Germany; sterilized at $120^{\circ} \mathrm{C}, 203 \mathrm{kPa}$ absolute pressure, $20 \mathrm{~min}$ ) for $30 \mathrm{~min}$ at $30^{\circ} \mathrm{C}$ with agitation. After centrifugation for $15 \mathrm{~min}$ and $800 \mathrm{~g}$ at $22^{\circ} \mathrm{C}, 25 \mathrm{ml}$ portions of the supernatant were frozen at $-25^{\circ} \mathrm{C}$. Aprotinin (Trasylol ${ }^{\circledR}$, Lot. No. 9011; $7139 \mathrm{KIU} / \mathrm{mg}$ polypeptide; Bayer AG, Wuppertal, Germany) was dissolved in sterile saline $(1 \mathrm{~g} / 30 \mathrm{ml})$ and applied at a concentration of $5 \mathrm{mg} / \mathrm{kg}$.

\section{Biochemical measurements}

The following quantities were determined in citrated plasma, bronchoalveolar lavage fluid or urine.

Sorbitol dehydrogenase ${ }^{3}$ ) according to l. c. (21), lactate dehydrogenase ${ }^{3}$ ), creatinine, bilirubin and urea, using test combinations (Boehringer, Mannheim, Germany). Total protein was determined by the biuret method, albumin by the bromocresol green method (22). Cell counting was performed in a Neubauer haemocytometer after staining with Tïrk's solution. The chemiluminescence response of citrated blood and isolated neutrophils was measured according to previously described methods $(19,22,23)$, in a sixchannel Biolumat LB 9505 (Berthold, Wildbad, Germany). Peak maximum values of zymosan-induced photon emission (counts/ min. 25000 neutrophils of isolated cell measurement; counts/min - 250000 neutrophils of blood measurement) were calculated as a measure of the total metabolic capacity/activity, non-induced photon emission (spontaneous) as a measure of in vivo activation by inflammatory mediators, and peak time values ( $\mathrm{min}$ ) as a measure of the surface receptor expression on neutrophils. Urine aprotinin concentration was measured according to $1 . c$. (24) and recalculated as units (kallikrein inhibitor units, KIU).

$\left.{ }^{2}\right)$ Aprotinin (Trasylol ${ }^{\circledR}$, Lot. No $9011 ; 7139 \mathrm{KIU} / \mathrm{mg}$ polypeptide) was kindly provided by Dr. F. Schumann, Bayer AG, Wuppertal, Germany

3) L-Iditol dehydrogenase (sorbitol dehydrogenase), L-Iditol : $\mathrm{NAD}^{+}$5-oxidoreductase (EC 1.1.1.15)

Kininogenin (Kallikrein) (EC 3.4.21.8)

Lactate dehydrogenase, $L$-lactate $: \mathrm{NAD}^{+}$oxidoreductase (EC 1.1.1.27)

Plasmin (EC 3.4.21.7) 


\section{Statistics}

Within- and between-group comparisons were performed on repeated measurements, using ANOVA ('STATISTICA' software program) with post-hoc comparisons (Tukey's HSD test). Significant within-group differences against baseline values are marked in the figures by two asterisks if $p<0.05$, while significant between group differences for corresponding time points are marked by one asterisk if $\mathrm{p}<0.05$.

Data in the figures are presented as the mean and the standard error of the mean $(\bar{x} \pm S E M)$.

\section{Results}

\section{Cardiac function and haemodynamics}

The cardiac index increase for the control group became significant at days $8-10$, whereas cardiac index for the aprotinin group remained in a normal range during the total observation period. There were significant differences between control and aprotinin groups at day 10 (fig. 1a).
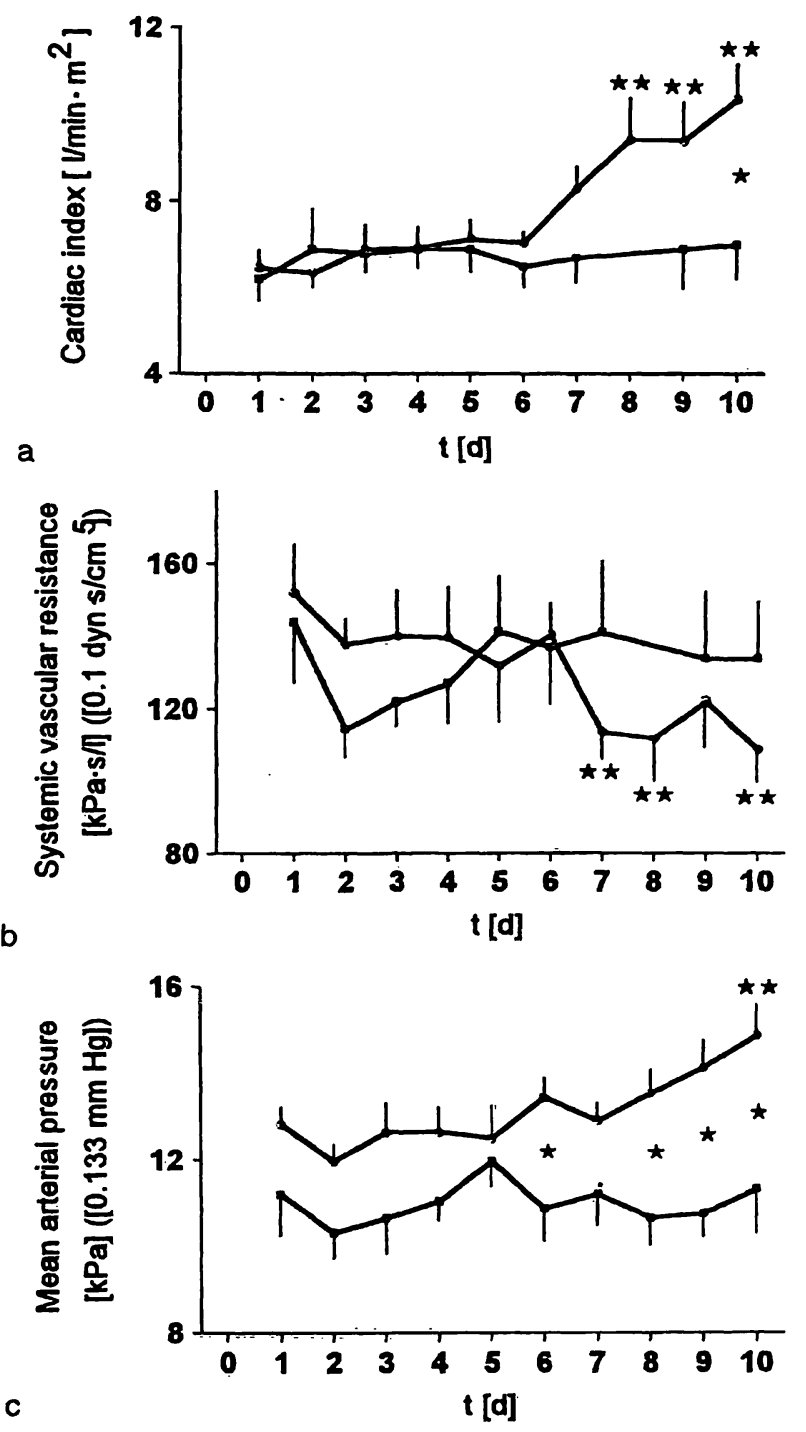

Fig. 1 Cardiac index (a), systemic vascular resistance (b), and mean arterial pressure (c) of multiple organ failure sheep in the control group (o; $n=10)$ and the aprotinin group $(n ; n=6)$ in the posttraumatic period.

Mean \pm SEM; ** $p<0.05$ values vs baseline; $* p<0.05$ control vs aprotinin group. Abscissa: time after haemorrhagic shock/intramedullary femoral nailing in days
Systemic vascular resistance decreased significantly for the control group at days 7,8 and 10 , whereas there was only an initial decrease of systemic vascular resistance for the aprotinin group at day 2 with a complete recovery till day 5 and with a constant level up to day 10 (fig. 1b).

Mean arterial pressure increased for the control group and remained unchanged for the aprotinin group. Between-group differences were significant at days 6 , 8-10 (fig. 1c).

\section{Pulmonary function}

Pulmonary arterial oxygen tension fell constantly till day 10 in both groups. There were significant differences between the groups at days 6 and 10 (fig. 2a). Pulmonary vascular resistance increased till day 10 in the control group, whereas there was no definite movement in the aprotinin group. Significant differences between the groups were observed at day 8 (fig. 2b). Mean pulmonary arterial pressure increased significantly at days 7-10 in the control group, but did not change in the aprotinin group during the total observation period. Significant differences between the groups became obvious at days 3-10 (fig. 3a). The epithelial lining fluid/ plasma ratio of albumin concentrations increased significantly after 6 and 10 days in the control group; there were significant differences between the groups after 10 days (fig. 3b).

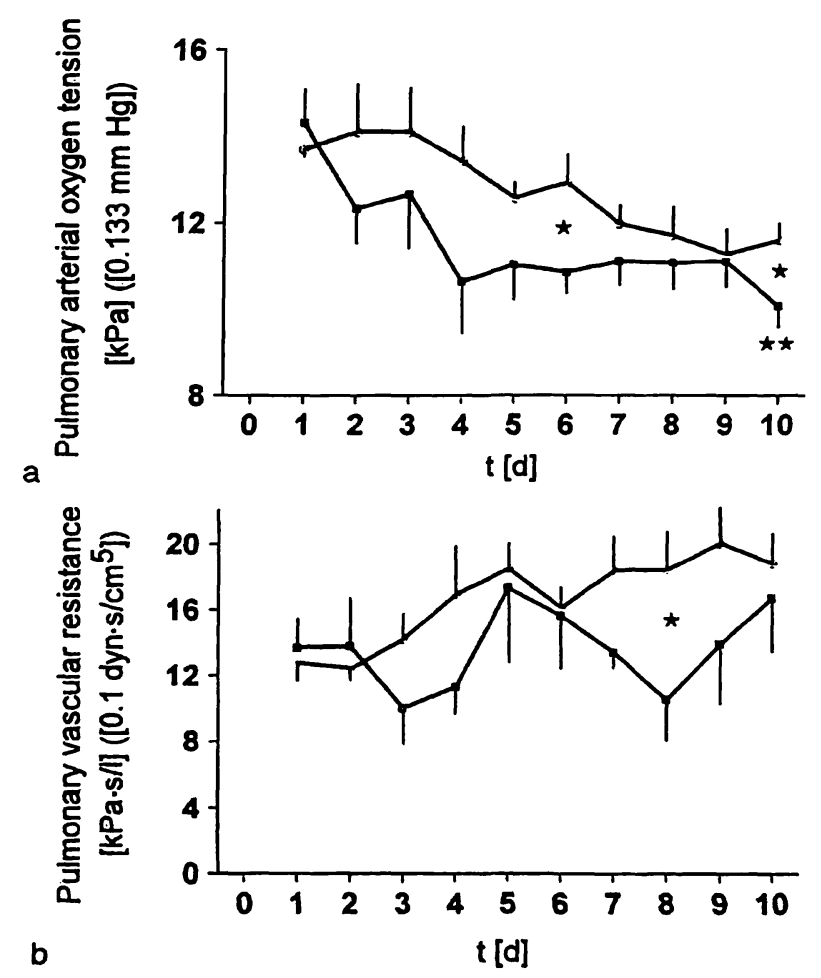

Fig. 2 Pulmonary arterial oxygen tension (a) and pulmonary vascular resistance (b) of multiple organ failure sheep without (o) and with ( $\mathbf{a})$ aprotinin therapy. 

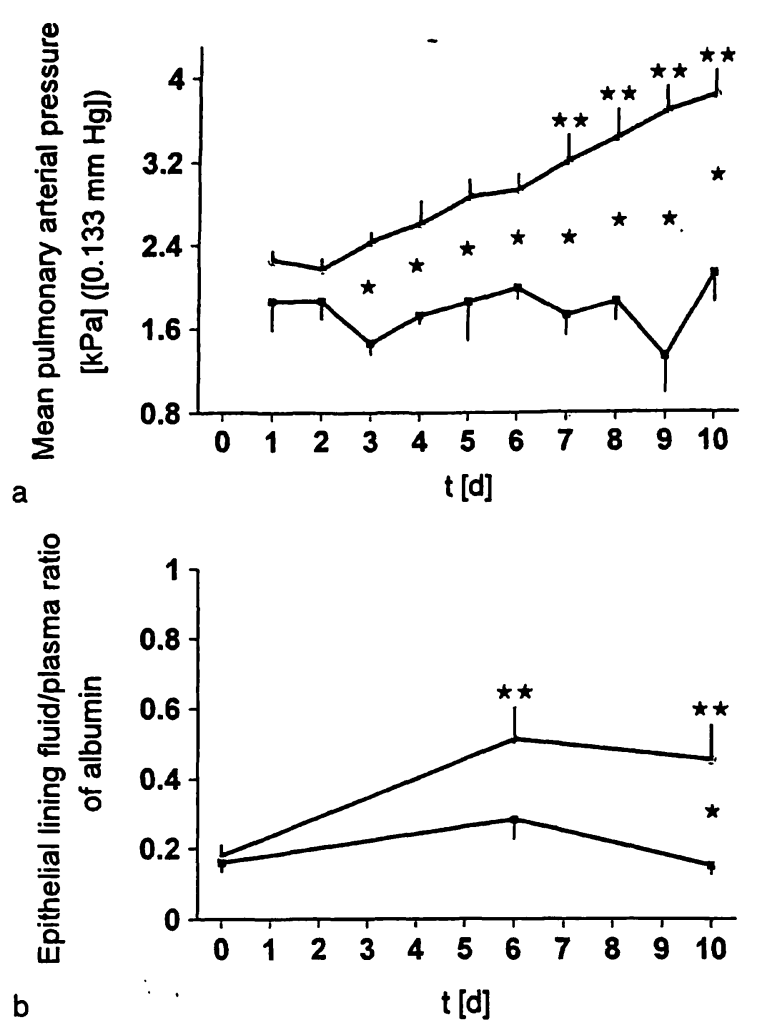

Fig. 3 Mean pulmonary arterial pressure (a) and epithelial lining fluid/plasma ratio (b) of albumin concentrations of multiple organ failure sheep without (o) and with ( $\square$ ) aprotinin therapy.
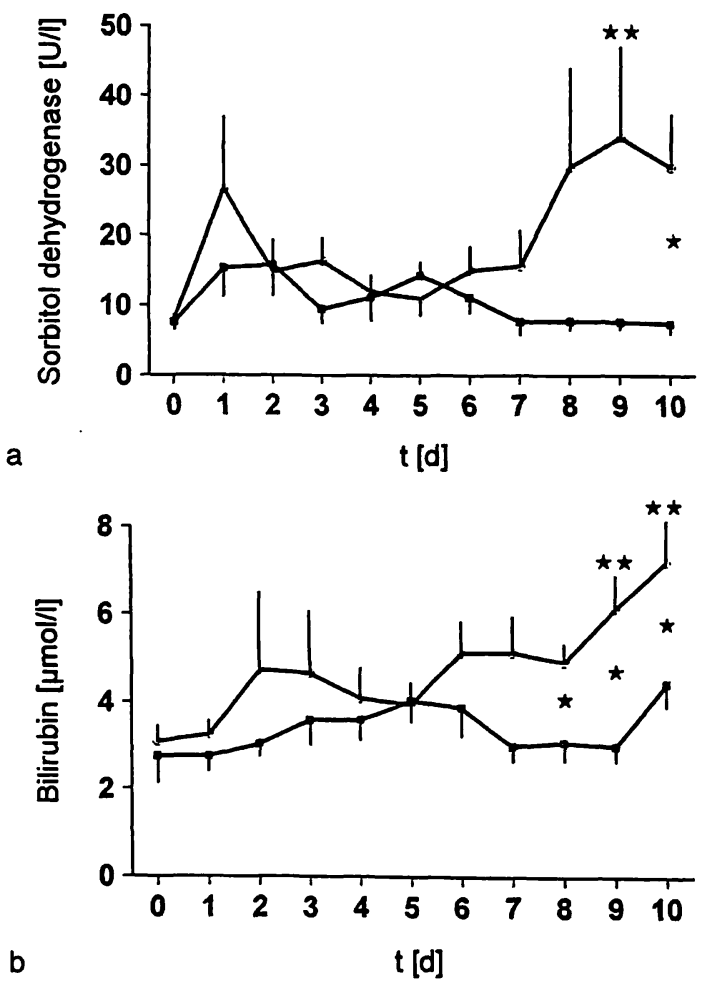

Fig. 4 Plasma sorbitol dehydrogenase activity (a) and plasma bilirubin concentration (b) of multiple organ failure sheep without $(\mathrm{O})$ and with (口) aprotinin therapy.

\section{Liver function}

After an initial reversible increase of the plasma sorbitol dehydrogenase of control animals at day 1 there was a late increase which became significant at day 9 , whereas aprotinin group values remained low for the total obser- vation period. Between-group differences were significant at day 10 (fig. 4a).

Plasma bilirubin concentrations in the control group indicated an increase that became significant at days 9 and 10 , whereas there was only a slight increase in the aprotinin group. The differences ibetween the groups were significant at days 8-10 (fig. 4b).

\section{Kidney function}

Creatinine clearance of the control group was normal till day 5 , then declined significantly at days 9 and 10 , whereas the aprotinin group indicated low values for the total period; between group differences were significant at day 5 (fig. 5a). The plasma urea concentration differed only at day 10 , in that the control group demonstrated non-significantly higher values (fig. 5b). The daily total amount of aprotinin eliminated with the urine peaked at day 3 and then fell despite further aprotinin administration (fig. 6).
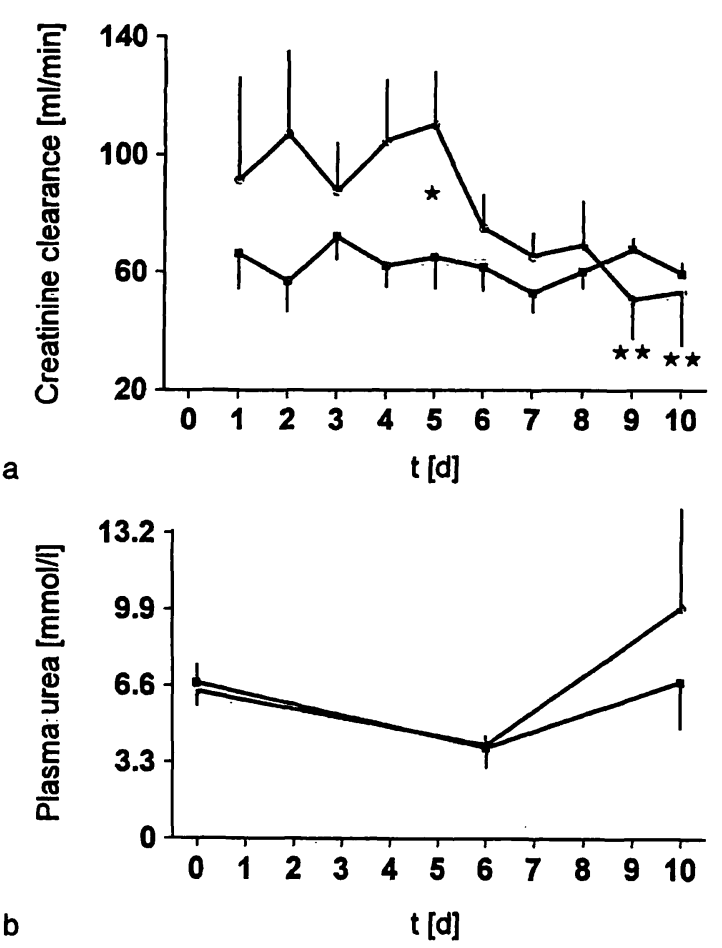

Fig. 5 Creatinine clearance (a) and plasma urea concentration (b)

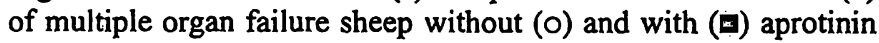
therapy.

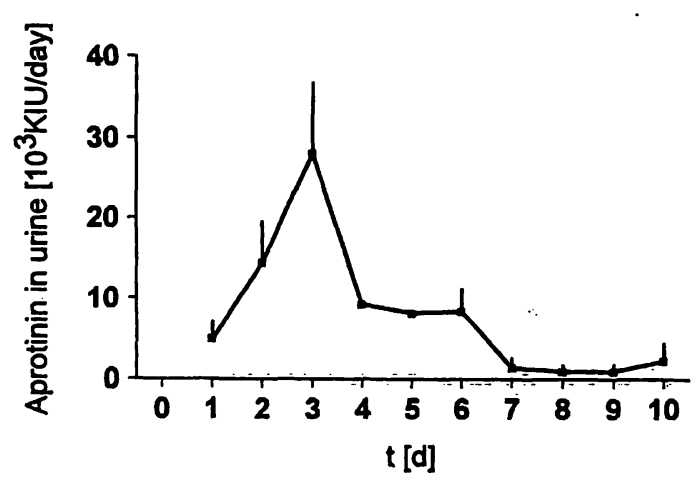

Fig. 6 Urinary aprotinin excretion of multiple organ failure sheep with aprotinin therapy. 


\section{General cytoprotection}

Plasma lactate dehydrogenase of the control group increased significantly at day 10 , whereas in the aprotinin group lactate dehydrogenase decreased steadily but non-significantly from the first day onwards. There were significant differences between the groups at days 9 and 10 (fig. 7).

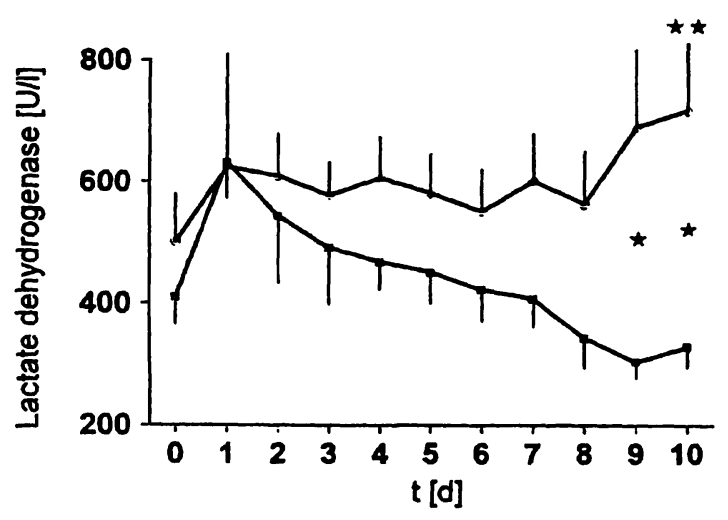

Fig. 7 Plasma lactate dehydrogenase activity of multiple organ failure sheep without $(0)$ and with $(\square)$ aprotinin therapy.
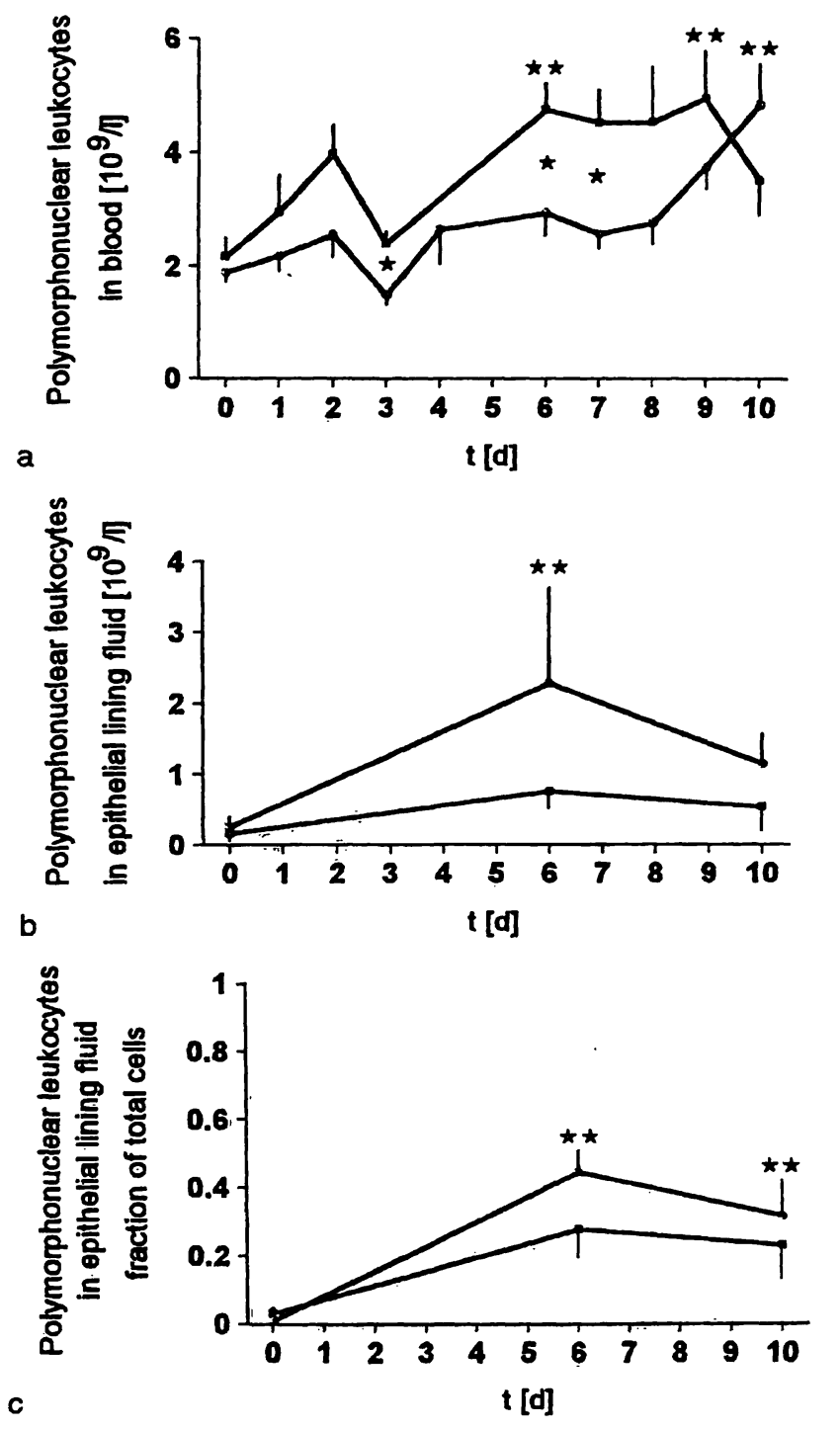

Fig. 8 Neutrophil count in blood (a) and in epithelial lining fluid $(b$ and $c$ ) of multiple organ failure sheep without $(0)$ and with $(\square)$ aprotinin therapy.
Neutrophil count and function in blood and epithelial lining fluid

Neutrophil count in blood increased significantly at day 10 for the control group and at days 6 and 9 for the aprotinin group; significant differences between the groups were observed at days 3, 6 and 7 (fig. 8a). Absolute neutrophil count in epithelial lining fluid increased significantly after 6 days for the control group, whereas aprotinin goup values remained low (fig. 8b). Calculation of the releative portions of alveolar neutrophils showed a significant increase after 6 and 10 days for the control group (fig. 8c).

The chemiluminescence response to zymosan by neutrophils isolated from the blood demonstrated a nearly unchanged course for the control group over the total observation period and a decrease of the aprotinin group till day 6 with subsequent recovery; the difference at day 6 between the two groups was significant (fig. 9a). The chemiluminescence response of neutrophils isolated from the epithelial lining fluid showed a significant decrease at day 6 for the control group, but there was no significant difference between control and aprotinin groups (fig. 9b). The chemiluminescence response difference of blood- and epithelial lining fluid-derived neutrophils (this difference indicates the loss of metabolic capacity of neutrophils during their capillary-interstitialalveolar passage and is a measure of the amount of reactive oxygen metabolites produced) increased significantly at day 6 for the control group, and there was a
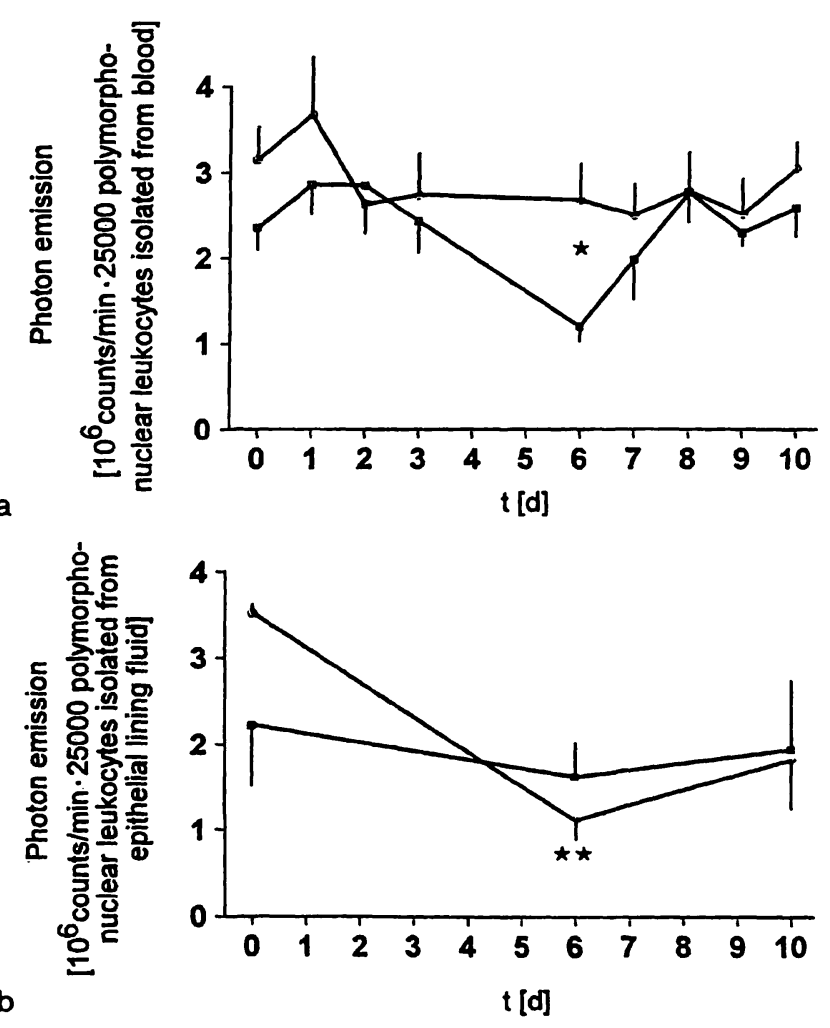

Fig. 9 Chemiluminescence response of blood-derived (a) and epithelial lining fluid-derived (b) neutrophils of multiple organ failure sheep without $(0)$ and with $(\mathbb{D})$ aprotinin therapy. 
significant difference between the two groups at day 6 (fig. 10). The spontaneous non-stimulated chemiluminescence response, indicating the in vivo production and stimulatory effect of inflammatory mediators, increased for both groups, but was significantly higher for the control group at day 6 (fig. 11a). The time taken to reach the photon emission maximum, indicating neutrophilic $\mathrm{C} 3 \mathrm{~b}$ receptor expression, decreased for the control group and increased for the aprotinin group; there were significant differences between the groups at days 2, 8 and 9 (fig. 11b).

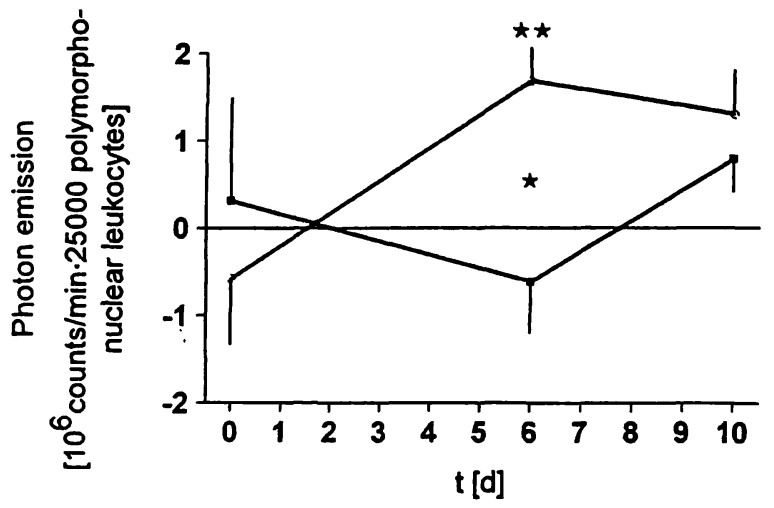

Fig. 10 Difference of chemiluminescence response of blood- and epithelial lining fluid-derived neutrophils of multiple organ failure sheep without (o) and with (घ) aprotinin therapy.
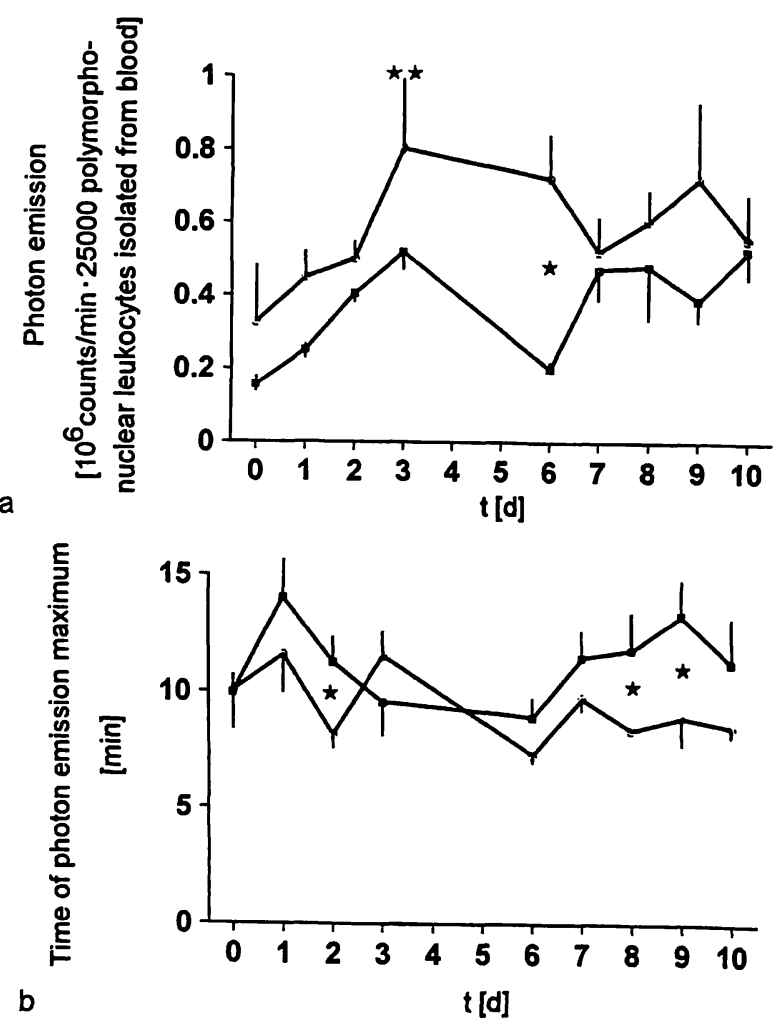

Fig. 11 Spontaneous chemiluminescence of blood-derived neutrophils of multiple organ failure sheep without (o) and with (⿴囗十) aprotinin therapy (a) and time to reach the maximum of photon emission response of blood-derived neutrophils of multiple organ failure sheep without (o) and with ( $($ ) aprotinin therapy (b).

\section{Discussion}

Extensive neutrophil and endothelial cell activation and interaction can result in the destruction of endothelial and parenchymal structures, and in organ failure. Important mediators of these reactions are reactive oxygen metabolites and proteinases which can induce each other's production, especially that of oxygen and nitric oxide radicals by blood cascade system proteinases. This can happen by direct or non-direct, i. e. complement-, fibrinolysis- or coagulation-associated reactions (fig. 12). The main target enzymes of aprotinin, plasmin and plasma kallikrein, are supposed to mediate neutrophil and endothelial cell activation. Therefore, the inhibition of these enzymes by aprotinin was expected to reduce inflammatory mediator production below the level that causes irreversible organ damage. To prove this assumption, we used an animal model of multiple organ failure which included the most important humoral and cellular activation steps shown after multiple injuries in humans $(9,10)$. In this model, sheep were subjected to haemorrhagic shock, intramedullary femoral nailing and repeated administration of endotoxin and zymosan-activated plasma for 5 days. The animals of the control group developed an irreversible sequential multiple organ failure between 6 and 10 days, as was demonstrated by monitoring the functional quantities of different organs (10). By a simultaneous treatment with aprotinin the significant alteration/impairment of several organ functions could be avoided, resulting in the healing of the experimentally induced multiple organ failure development. Judged by different functional quantities, two out of five organs/systems monitored demonstrated an absolute and significant improvement.

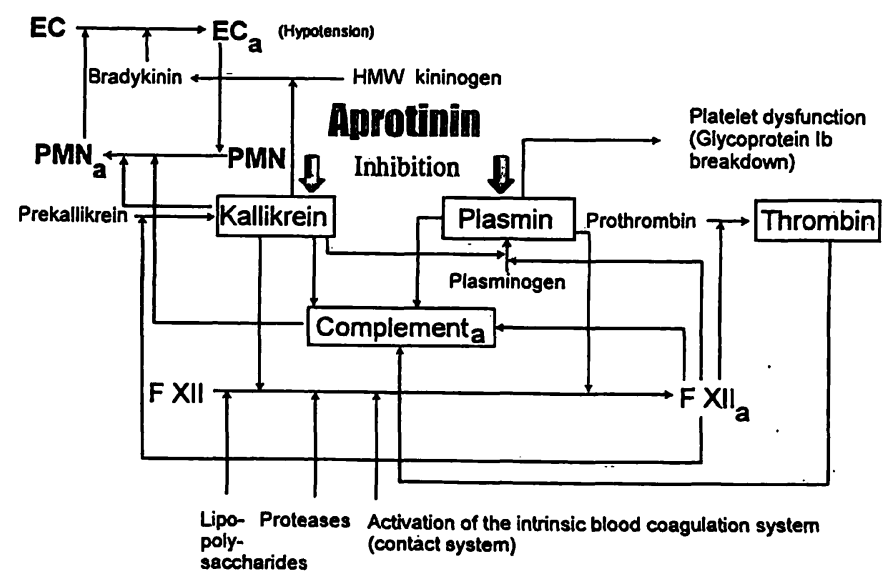

Fig. 12 Inhibition by aprotinin of plasmin- and kallikrein-associated pathways.

$\mathrm{EC}=$ endothelial cell

$\mathrm{EC}_{\mathrm{a}}=$ activated endothelial cell

PMN = polymorphonuclear leukocyte

$\mathrm{PMN}_{\mathrm{a}}=$ activated polymorphonuclear leukocyte

$\mathrm{HMW}$ kininogen $=$ high molecular weight kininogen

F XII = Hagemann factor

$\mathrm{F} \mathrm{XII}_{\mathrm{a}}=$ activated Hagemann factor 
Cardiac index, systemic vascular resistance and mean arterial pressure indicated a significant normalisation of cardiac function and haemodynamics.

Bilirubin as a marker of excretory and detoxicative liver functions and sorbitol dehydrogenase as a marker of hepatocyte membrane integrity indicated a significant improvement of liver function.

Furthermore, a general cytoprotective effect of aprotinin presumably comprising all organs was observed by measuring plasma lactate dehydrogenase.

Concerning the lung, there was no unique behaviour of functional quantities. Three of them normalised with aprotinin therapy (pulmonary vascular resistance, mean pulmonary arterial pressure, epithelial lining fluid/ plasma ratio of albumin as a direct biochemical marker of the lung capillary permeability damage (25)), whereas one of them remained pathological (pulmonary arterial oxygen tension). Therefore, the term 'improvement of lung function' should be restricted to defined lung quantities considered in this context.

The only organ without perceptible amelioration by aprotinin was the kidney. Previously described renal effects of aprotinin (26) were also found in the depression of the creatinine clearance for the total observation period without tendency to improve. In contrast, Cumming et al. found an aprotinin-induced improvement of the renal function in an acute sheep model of septic shock (18). This may be explained by the difference of the models used. The plasma urea concentration fell only insignificantly at day 10 , and the aprotinin excretion into the urine decreased from day 3 onwards, despite continuous administration of aprotinin, indicating the early impairment of the renal function.

The pathophysiology of this type of induced organ failure seems to be associated with neutrophil dysfunction $(3,8,27)$ and is assumed to be identical in different organs. But, because of its unique anatomy, the lung in particular offers the experimental design for easily isolating and investigating neutrophils before (capillary neutrophils) and after (alveolar neutrophils) they have participated in the destructive processes of organ failure during their capillary-interstitial-alveolar transition.

Thus, the increased alveolar influx of neutrophils, already previously observed $(19,28)$, indicated the participation of neutrophils, chemotaxins like IL-8 or C5a (29) and reactive oxygen metabolites (30). This neutrophil influx seemed to be reduced by the aprotinin-induced inhibition of plasmin- and kallikrein-associated chemotaxin formation (fig. 12). The aprotinin-induced reduction of inflammatory mediator production was reflected by the reduction of the spontaneous photon emission of isolated neutrophils which had experienced less in vivo priming by different amounts and/or types of inflammatory mediators $(31,32)$. This was confirmed by the aprotinin-mediated reduction of in vivo neutrophil activation, measured by the increase of the time taken to attain the photon emission maximum of in vitro zymosan-activated neutrophils, representing a reciprocal measure of in vivo $\mathrm{C} 3 \mathrm{~b}$ receptor expression by inflammatory mediator-induced activation (23).

The difference between the in vitro-induced reactive oxygen metabolite production of simultaneously isolated blood neutrophils and epithelial lining fluid neutrophils was used as an index of lung tissue damage by neutrophils during their passage from the capillary into the alveolus. For animals of the control group, which developed multiple organ failure, this difference was significantly higher in comparison with aprotinin-treated animals which did not develop multiple organ failure.

The greater extent of control neutrophil priming by a higher amount and/or more effective inflammatory mediators (as observed by the higher in vitro response of control neutrophils) enables neutrophils in vivo and in vitro to increase their reactive oxygen metabolite production upon secondary stimulation and to produce a higher degree of tissue damage. Since aprotinin prevents the plasmin- and kallikrein-associated production of priming inflammatory mediators, it contributes to the reduction of reactive oxygen metabolites produced by secondary stimulation and to the reduction of tissue destruction.

In summary, by the inhibition of plasmin- and kallikreinmediated pathways, aprotinin improved the function of several injured organs, except kidney, and thus, prevented at least partially the development of an experimentally induced multiple organ failure in sheep.

\section{Acknowledgements}

The experiments of the study were performed with the technical assistance of $M$. Funck, K. Krumm, B. Lueken, A. Oettl and G. Schweitzer.

\section{References}

1. Godin C, Caprani A, Dufaux J, Flaud P. Interactions between neutrophils and endothelial cells. J Cell Science 1993; $106: 441-52$.

2. Rinaldo JE, Christman JW. Mechanisms and mediators of the adult respiratory distress syndrome. Clin Chest Med 1990; 11(4):621-32. 
3. Gee MH, Albertine KH. Neutrophil-endothelial cell interactions in the lung. Annu Rev Physiol 1993; 55:227-48.

4. Lucchesi BR. Complement activation, neutrophils, and oxygen radicals in reperfusion injury. Stroke 1993; 24(12) Suppl $\mathrm{I}: 1-41-7$

5. Lefer AM, Lefer DJ. Pharmacology of the endothelium in ischemia-reperfusion and circulatory shock. Annu Rev Pharmacol Toxicol 1993; 33:71-90.

6. St. John RC, Dorinsky PM. Immunologic therapy for ARDS, septic shock, and multiple-organ failure. Chest 1993; 103:932-43.

7. Brigham $\mathrm{KL}$. Oxidant stress and adult respiratory distress syndrome. Eur Respir J 1993; 3 Suppl 11:482s-4s.

8. Dorinsky PM, Gadek JE. Multiple organ failure. Clin Chest Med 1990; 11(4):581-91.

9. Grotz M, Regel G, Dwenger A, Pape HC, Hainer C, Vaske R, et al. Ein standardisiertes Großtiermodell zum Multiorganversagen (MOV) nach schwerem Trauma. Unfallchirurg 1995; 98:63-71.

10. Grotz M, Remmers D, Dwenger A, Pape HC, Hainer C, Regel G. A standardized sheep-model for multiple organ failure after severe trauma. Clin Int Care 1994; 5 Suppl:31.

11. Divenger A, Pape HC, Bantel C, Schweitzer G, Krumm K, Grotz $M$, et al. Ascorbic acid reduces the endotoxin-induced lung injury in awake sheep. Eur J Clin Invest 1994; 24:22935 .

12. Jepsen $\mathrm{S}$, Herlevsen $\mathrm{P}$, Knudsen $\mathrm{P}$, Bud MI, Klausen N-O. Antioxidant treatment with $\mathrm{N}$-acetylcysteine during adult respiratory distress syndrome: a prospective, randomized, placebo-controlled study. Crit Care Med 1992; 20 (7):918-23.

13. Marzi I, Bühren V, Schüttler A, Trentz $O$. Value of superoxide dismutase for prevention of multiple organ failure after multiple trauma. J Trauma 1993; 35 (1):110-20.

14. Redens TB, Leach WJ, Bogdanoff DA, Emerson TE. Synergistic protection from lung damage by combining antithrombin III and al-proteinase inhibitor in the E. coli endotoxemic sheep pulmonary dysfunction model. Circ Shock 1988; 26:15-26.

15. Schwartz RS, Bauer KA, Rosenberg RD, Kavanaugh EJ, Davies DC, Bogdanoff DA. Clinical experience with antithrombin III concentrate in treatment of congenital and acquired deficiency of antithrombin III. Am J Med 1989; 87 Suppl 3B:53S-60S

16. Siebeck M, Fink E, Weipert J, Jochum M, Fritz H, Spannagl $\mathrm{M}$, et al. Inhibition of plasma kallikrein with aprotinin in porcine endotoxin shock. J Trauma 1993; 34 (2):193-8.

17. Huang H, Ding W, Su Z, Zhang W. Mechanism of the preserving effect of aprotinin on platelet function on its use in cardiac surgery. J Thorac Cardiovasc Surg 1993; 106:11-8.

18. Cumming AD, Nimmo GR. Hemodynamic, renal, and hormonal actions of aprotinin in an ovine model of septic shock. Crit Care Med 1992; 20:1134-9.

19. Dwenger A, Regel G, Ellendorff B, Schweitzer G, Funck M, Limbrock $\mathrm{H}$, et al. Alveolar cell pattern and chemiluminescence response of neutrophils and alveolar macrophages in sheep after endotoxin injection. J Clin Chem Clin Biochem $1990 ; 28: 163-8$.
20. Pape HC, Dwenger A, Regel G, Schweitzer G, Jonas M, Remmers $D$, et al. Pulmonary damage after intramedullary femoral nailing in traumatized sheep - is there an effect from different nailing methods? J Trauma 1992; 33 (4):574-81.

21. Gerlach U. Sorbitol dehydrogenase. In: Bergmeyer HU, editor. Methods of enzymatic analysis. Weinheim: Verlag Chemie, 1983; 3:112-7.

22. Dwenger A, Schweitzer G, Regel G. Bronchoalveolar lavage fluid and plasma proteins, chemiluminescence response and protein contents of polymorphonuclear leukocytes from blood and lavage fluid in traumatized patients. $J$ Clin Chem Clin Biochem 1986; 24:73-88.

23. Dwenger A, Regel G, Schweitzer G. Pathomechanisms of the adult respiratory distress syndrome (ARDS). Chemiluminescence analysis of polymorphonuclear leukocytes. Fresenius $\mathbf{Z}$ Anal Chem 1986; 324:360-1.

24. Müller-Esterl W. Aprotinin-enzyme-linked immunosorbent assay. In: Bergmeyer HU, editor. Methods of enzymatic analysis. 3rd ed. Weinheim: Verlag Chemie, 1983; 12:246-56.

25. Dwenger A, Schweitzer G, Funck M. Plasma and bronchoalveolar lavage fluid proteins as markers of increased lung permeability in ARDS as a result of multiple trauma. In: Sturm JA, editor. Adult respiratory distress syndrome - an aspect of multiple organ failure. Berlin-Heidelberg: Springer-Verlag, 1991:215-29.

26. Kauker ML. Renal effects of aprotinin, a kallikrein inhibitor in rats in saline diuresis (42002). Proc Soc Exper Biol Med $1985 ; 178: 222-6$.

27. Tate RM, Repine JE. Neutrophils and the adult respiratory distress syndrome. Am Rev Respir Dis 1983; 128:552-9.

28. Weiland JE, Davis WB, Holter JF, Mohammed JR, Dorinsky PM, Gadek JE. Lung neutrophils in the adult respiratory distress syndrome. Am Rev Respir Dis 1986; 133:218-25.

29. Jorens PG, Van Damme J, De Backer W, Bossaert L, De Jongh $\mathrm{RF}$, Herman AG, et al. Interleukin 8 (IL-8) in the bronchoalveolar lavage fluid from patients with the adult respiratory distress syndrome (ARDS) and patients at risk for ARDS. Cytokine 1992; 4 (6):592-7.

30. Petrone WF, English DK, Wong K, McCord JM. Free radicals and inflammation: superoxide-dependent activation of a neutrophil chemotactic factor in plasma. Proc Natl Acad Sci USA 1980; 77:1159-63.

31. Dwenger A, Schweitzer G, Funck M. Lipopolysaccharide-dependent enhancement of adherence-mediated chemiluminescence response of polymorphonuclear leukocytes. J Biolumin Chemilumin 1988; 2:35-9.

32. Tortorella C, Ottolenghi A, Capobianco V, Jirillo E, Antonaci S. Effects of lipopolysaccharide, interferon- $\alpha$ and/or interferon- $\gamma$ priming on polymorphonuclear cell respiratory burst kinetics. Med Sci Res 1993; 21:217-8.

\section{Received May 20/November 23, 1995}

Corresponding author: Dr. rer. nat. Alexander Dwenger, Institut für Klinische Biochemie, Medizinische Hochschule Hannover, D-30623 Hannover, Germany 